\title{
Comparación entre Oracle BPM Y JBPM en la optimización de un proceso de admisiones
}

\author{
Comparison between Oracle BPM and JBPM in the Admission \\ Process Optimization
}

Fecha de Recepción: 2013-05-31

Fecha de Aprobación: 2013-06-21
Jorge Leonardo Camargo Cuervo* Edgar Fernando Suárez Mendoza** Javier Antonio Ballesteros Ricaurte ${ }^{* * *}$

\section{Resumen}

Se presenta el procedimiento seguido para evaluar y comparar dos suites en la Gestión por Procesos de Negocio: Oracle BPM y JBPM; procedimiento que se basó en la ponderación y gradación de las características Implementación, Integración, Desempeño, Escalabilidad y Documentación de cada suite en el caso de automatizar el proceso de Admisiones de la Oficina de Registro Académico de la Universidad Pedagógica y Tecnológica de Colombia, cuya complejidad y transversalidad a toda la universidad lo señalaron como el más apropiado para el proyecto. Para lograr el objetivo de este

\section{Abstract}

It's shown the procedure to evaluate and compare two suites in the Business Processes Management, both Oracle BPM and JBPM, which allows achieving visible results on the project through the weighing and gradation of the following characteristics: Implementation, integration, performance, scalability and documentation. The tools comparison is based on a study to automate the students admissions process of the Academic Registration Office, in the Pedagogic and Technologic University of Colombia, taking into account that this is a transversal process for all the university.

\footnotetext{
* Universidad Pedagógica y Tecnólogica de Colombia (Colombia). jorgeleonardo.camargo@uptc.edu.co

** Universidad Pedagógica y Tecnólogica de Colombia (Colombia).edgar.suarez@uptc.edu.co

*** M.Sc. Universidad Pedagógica y Tecnólogica de Colombia (Colombia). javier.ballesteros@uptc.edu.co
} 
trabajo se utilizó la metodología SCRUM, que permite un desarrollo ágil y eficaz.

Palabras clave: Gestión de Procesos de Negocio, BPM, Oracle, Oracle BPM, JBPM, Optimización de procesos organizacionales.
To achieve this paper's goal, it was used the SCRUM methodology which allows an agile, fast and effective development.

Keywords: Business Process Management, BPM, Deployment, Agility, Process. Optimizing Organizational Process. 


\section{INTRODUCCIÓN}

La optimización de procesos organizacionales ha venido tomando auge, en especial por la implementación de tecnologías como Gestión o Administración de Procesos de Negocio (BPM por su sigla en inglés); por tal motivo resulta indispensable tener conocimientos especialmente prácticos acerca del uso de esta tecnología, mediante las distintas suites de BPM. De este modo, a continuación se presentan los resultados de una implementación hecha con BPM usando dos herramientas: Oracle BMP y JBMP; en dicha comparación se tuvieron en cuenta cuatro aspectos importantes: Implementación, Desempeño, Integración y Escalabilidad, y la Documentación, que durante el desarrollo del proyecto fue tomando importancia como aspecto para evaluar y que terminó siendo fundamental en los resultados finales; tomando estas características se propugna fortalecer los conocimientos sobre el desarrollo de aplicaciones basadas en procesos.

En la comparación de las herramientas, para el caso particular de la investigación, se selecciona el proceso de Admisión de la Universidad Pedagógica y Tecnológica de Colombia, que con su complejidad y transversalidad se convierte en el candidato apropiado para realizar el proceso de automatización. El análisis realizado a cada paso en el proceso de admisiones brindó la información necesaria para evaluar las herramientas en cada característica; los funcionarios también fueron claves en cada revisión, debido a que su conocimiento y experiencia sirvieron para la automatización y puesta en marcha del proceso en cada suite.

Finalmente, se plasman una serie de conclusiones producto del análisis realizado a las herramientas evaluadas, y que en general propenden al desarrollo del conocimiento en esta importante área de las tecnologías de la información y la comunicación.

\section{Contextualización}

Con el fin de proveer una base para la comprensión del trabajo realizado se presentan a continuación los conceptos clave para un completo entendimiento del caso de estudio abordado para esta investigación.

\section{A. Proceso de negocio}

El proceso de negocio hace referencia a una serie de tareas organizacionales que en conjunto y a partir de unos insumos base dan como resultado un producto o un servicio para un cliente o usuario final [1]; como insumos se entiende cualquier recurso material o inmaterial necesario para proveer el servicio final.

\section{B. Business Process Management (BPM)}

La Gestión de Procesos de Negocio, o Business Process Management (BPM), se define como una metodología que busca optimizar los procesos de una empresa, utilizando herramientas y tecnologías para diseñar, representar, analizar y controlar procesos de negocio operacionales [1]; estos procesos deben ser modelados, automatizados, integrados y monitoreados de forma continua. BPM trabaja a partir de un marco documental que permite crear, modificar y gestionar procesos y facilitar el acceso a ellos en cualquier momento [2].

\section{Business Process Modeling Notation (BPMN)}

Para el modelamiento de procesos, BPM provee una notación gráfica que permite la implementación gráfica completa de procesos de negocio o, bien, es un formato de flujo de trabajo. Se buscó una notación que sea de fácil entendimiento y comprensión de todos los usuarios [3]; esta notación se basa en una técnica de diagramas de flujo adaptados para la creación de modelos gráficos de las operaciones de procesos de negocio. 


\section{Oracle BPM}

Oracle presenta una suite para trabajar la metodología BPM, esto con el fin de buscar una mayor eficiencia, visibilidad y agilidad en el momento de entregar productos líderes para la gestión de procesos de negocio [4]. Oracle, por medio de su suite Oracle BPM, busca unificar procesos que tienen una aplicación existente, buscando simplificar el modelado de procesos, la ejecución y la interacción de los participantes; estas características se pueden observar en la tabla 1.

\section{TABLA 1 \\ Características Oracle BPM [5]}

\begin{tabular}{|l|l|}
\hline \multicolumn{1}{|c|}{ Característica } & \multicolumn{1}{c|}{ Beneficio } \\
\hline $\begin{array}{l}\text { Unificación BPM 2.0, BPEL, reglas y flujos de trabajo del } \\
\text { motor }\end{array}$ & $\begin{array}{l}\text { Utiliza un conjunto de infraestructura para todos los requisitos } \\
\text { del proceso }\end{array}$ \\
\hline Ejecución nativa BPM 2.0 & Ejecuta los procesos sin necesidad de traducción \\
\hline Integración con Fusion Middleware y Microsoft Office & Interacción con herramientas de escritorio familiares \\
\hline Catálogo de objetos y servicios & Permite a la empresa reutilizar los activos a través de procesos \\
\hline Oracle BPM Studio & Permite modelar y crear aplicaciones basadas en procesos \\
\hline Oracle Process Composer & $\begin{array}{l}\text { Permite crear y personalizar los procesos de negocio en una } \\
\text { herramienta web }\end{array}$ \\
\hline Zero-code Process Development Wizards & Reduce la complejidad de las implementaciones de procesos \\
\hline BPMN 2.0 y modelado BPEL & Utiliza los estándares para la diagramación de modelos BPM \\
\hline Simulación, análisis de procesos y presentación de informes & $\begin{array}{l}\text { Evalúa las decisiones de los procesos con datos reales de la } \\
\text { empresa }\end{array}$ \\
\hline $\begin{array}{l}\text { Espacio para todos los procesos, modelados e instancias de } \\
\text { un solo proceso }\end{array}$ & $\begin{array}{l}\text { Colaboración de la suite con todos los participantes de un } \\
\text { proceso }\end{array}$ \\
\hline Planificación de procesos no estructurados & Permite la fácil gestión de procesos dinámicos \\
\hline Guías de procesos de negocio & $\begin{array}{l}\text { Agiliza la comunicación de flujo del proceso y la situación de } \\
\text { la empresa }\end{array}$ \\
\hline
\end{tabular}

\section{E. JBPM}

Es una herramienta Open Source que permite modelar procesos de negocio para el posterior despliegue y gestión; facilita el entendimiento e implementación de BPM, puesto a que su forma de trabajo se hace de manera simple y concisa, logrando que los usuarios tengan un entendimiento claro y fácil de esta herramienta [6]. En la tabla 2 se presentan las características que se tienen en cuenta en el proceso.

TABLA 2

CARACTERÍSTICAS JBPM [7]

\begin{tabular}{|l|l|}
\hline \multicolumn{1}{|c|}{ Característica } & \multicolumn{1}{c|}{ Beneficio } \\
\hline $\begin{array}{l}\text { Avances en el proceso de diseño basado en la } \\
\text { Web }\end{array}$ & $\begin{array}{l}\text { Soporte para diferente tipos de nodos, validación visual, apoyo en la } \\
\text { migración de procesos, etc. }\end{array}$ \\
\hline AS7 JBoss & $\begin{array}{l}\text { AS7 es el nuevo servidor de aplicaciones que va a soportar todas las insta- } \\
\text { laciones en JBPM. }\end{array}$ \\
\hline Núcleo del motor & Da soporte para la persistencia de múltiples bases de datos. \\
\hline Instalación & Soporte para JBoss AS7. \\
\hline Web de diseñador & Posibilidad de ver los procesos en FEDER, JSON, PDF, PNG y formatos SVG. \\
\hline Servicios de tareas humanas & Permite la validación de usuarios utilizando la gestión de grupos de trabajo. \\
\hline Consola JBPM & $\begin{array}{l}\text { Permite la sincronización de los procesos con el repositorio seleccionado y } \\
\text { la creación de informes BIRT. }\end{array}$ \\
\hline Plug-in Eclipse & Se trabaja bajo la perspectiva de Eclipse. \\
\hline
\end{tabular}




\section{F. SCRUM}

Es una metodología para la gestión de proyectos; se caracteriza por su agilidad para elevar al máximo la productividad de un equipo. Una característica importante de esta metodología es que acaba la burocracia o jerarquía dentro de un grupo de trabajo [8]. Entre sus grandes características se encuentra que es un modelo de carácter adaptable más que predictivo; se orienta a las personas, más que a los procesos, e implementa una estructura basada en interacciones y revisiones [9].

\section{Desarrollo}

Para llevar a cabo la investigación propuesta se inicia con la identificación del caso de estudio apropiado para realizar la implementación de BPM. El aspecto fundamental que se tiene en cuenta para identificar procesos candidatos a automatización con BPM es la transversalidad que tenga dentro de la organización.

\section{A. Metodología para la automatización}

Para el caso de estudio seleccionado se opta por utilizar una metodología de desarrollo ágil que cumpla con las características de rapidez y eficiencia que requiere BPM. De esta forma, se selecciona la metodología SCRUM, que para el caso de estudio planteó el desarrollo de tres Sprint, según la Fig. 1.
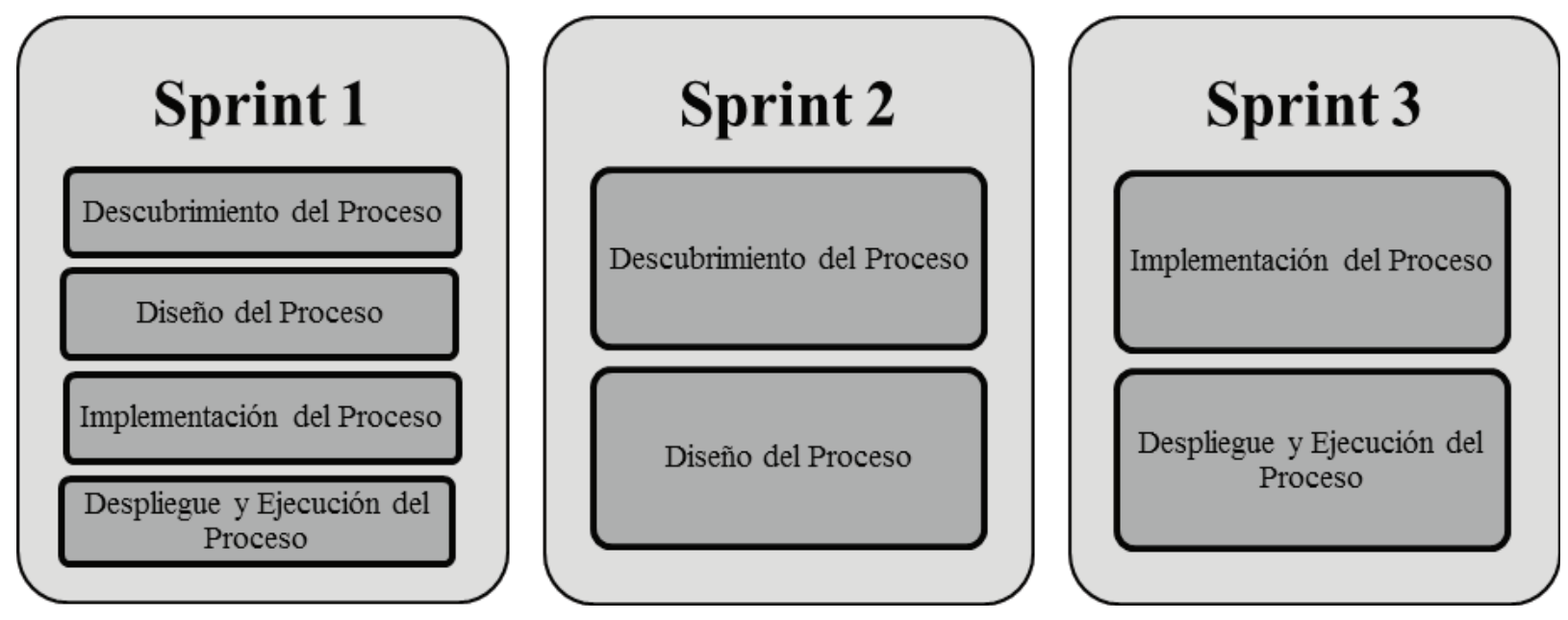

Fig. 1. Planeación Metodología SCRUM

Para el primer Sprint se realiza un proceso plano, que no contempla interacción con el usuario, pero es importante por el hecho de ser la base para futuras ejecuciones del proceso, ya que se definen las configuraciones básicas para este.

Para el segundo y tercer Sprint se realiza un diseño más detallado del proceso y se implementan las pantallas de interacción con el usuario, lo que complementa en su totalidad el desarrollo del proceso.

\section{B. Diseño general del proceso}

La metodología plantea como primer paso el desarrollo de reuniones con los dueños del proceso, para obtener la información necesaria para el diseño de este en notación BPMN (ver Fig. 2). 


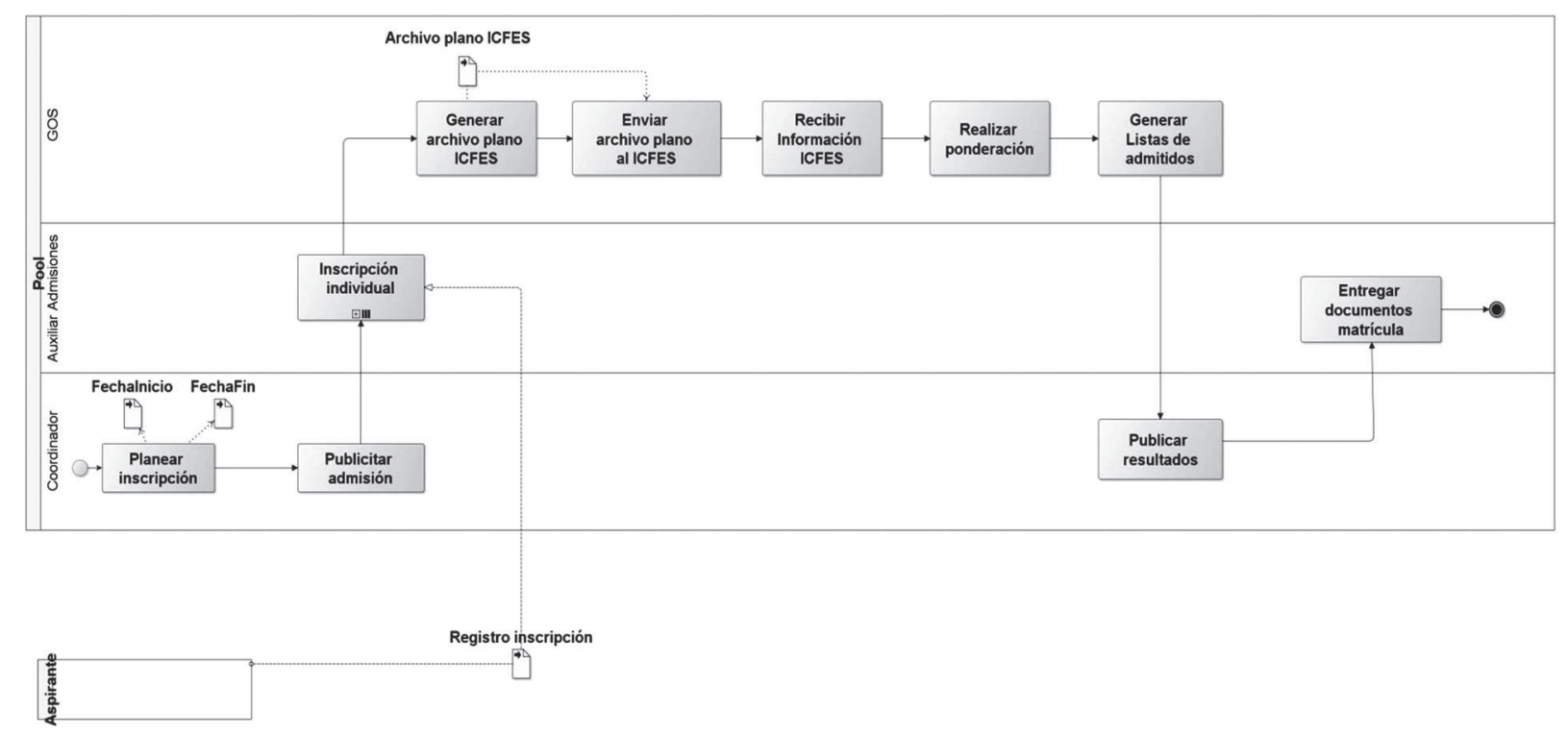

Fig. 2. Diseño general del proceso

\section{Implementación del proceso en Oracle BPM}

Oracle BPM ofrece, en buena medida, los componentes de BPMN, por lo que las modificaciones realizadas al proceso no son

muy grandes. Dentro de los componentes que ofrece se encuentran las tareas manuales, lo cual evita que se deba modificar el proceso original en cuanto a este aspecto (ver Fig. 3).

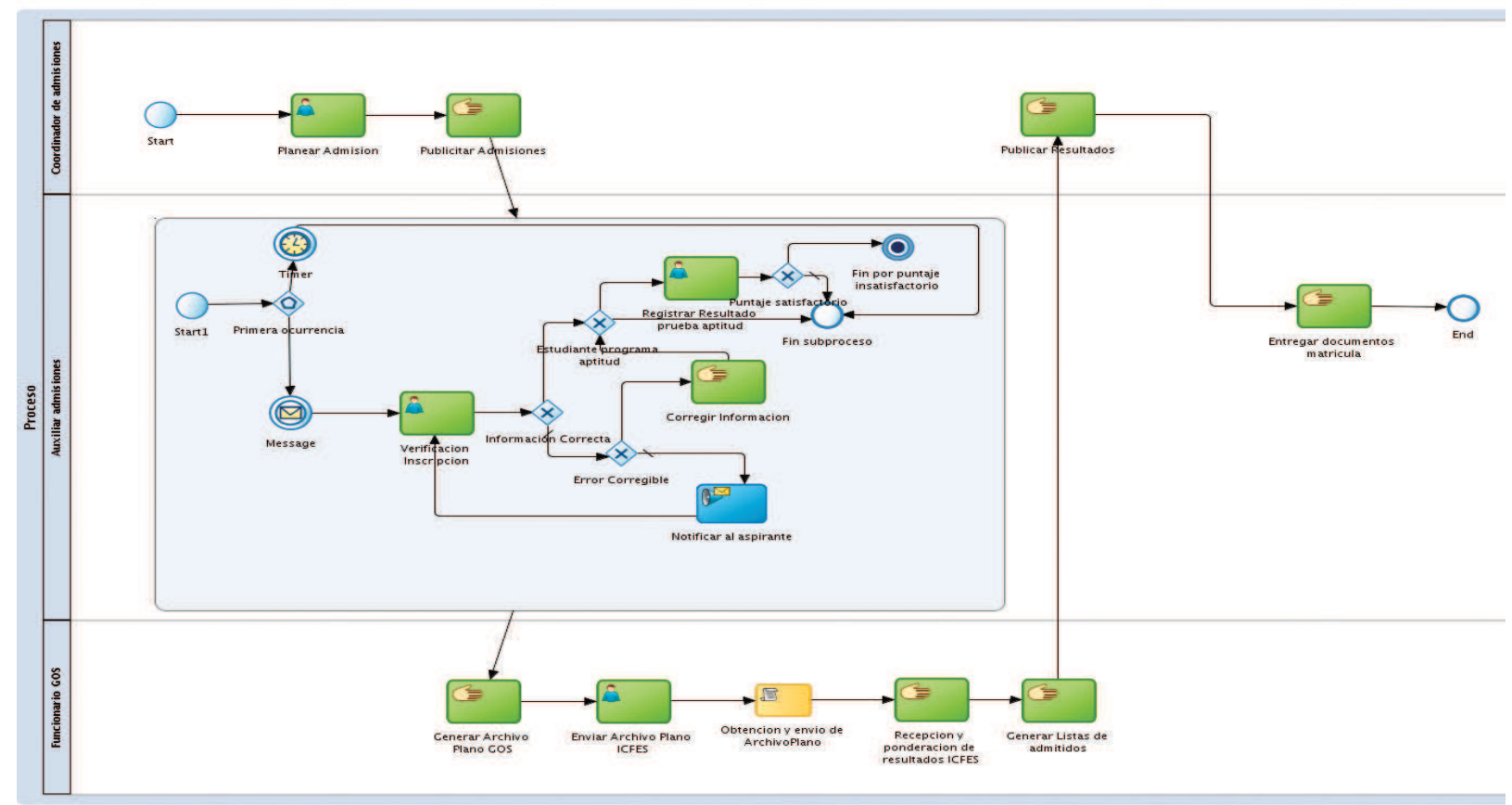

Fig. 3. Implementación del proceso en Oracle 
Por otra parte, dentro de las compuertas que ofrece Oracle BPM se encuentra la basada en eventos ("Primera Ocurrencia"), que fue una de las que se contempló en el diseño original. Para esa misma sección del proyecto se pudo contar con un evento de captura de tiempo que evalúa para el caso particular la fecha final de inscripción; del mismo modo, un evento de recepción de mensaje permitió modelar la sección que permite evaluar el momento en que se realiza una inscripción para ser verificada.
La suite cuenta con múltiples actividades de notificación, lo que facilitó el modelado de la sección en la que se notifica al aspirante de la presencia de un error en su inscripción.

\section{Implementación del proceso en JBPM}

A diferencia de la suite de Oracle, JBPM ofrece menos componentes para la implementación de los procesos; para el caso de esta suite fue necesario realizar ciertos ajustes al proceso, los cuales garantizan la ejecución eficaz (ver Fig. 4).

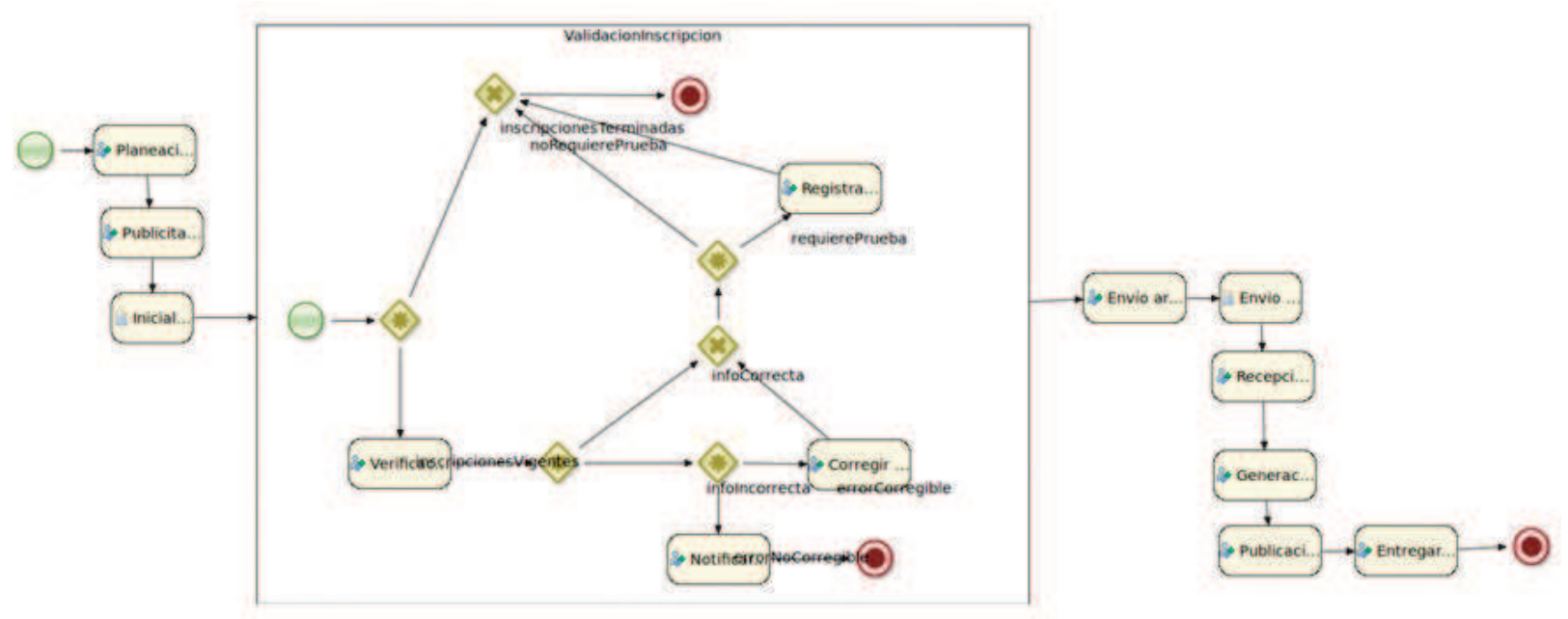

Fig. 4. Implementación del proceso en JBPM

Uno de los componentes que no provee JBPM es la actividad manual, que para el caso del proceso es muy común; en lugar de esto, se debió modificar el proceso para que las tareas manuales del diseño original fueran tareas humanas en este caso, con la diferencia de que las pantallas no presentan ninguna entrada, solamente un botón que permite concluir la tarea cuando sea necesario.

La suite tampoco provee compuertas basadas en eventos; por este motivo se modificó el proceso para que con una compuerta tipo OR se validara si la fecha final de inscripción aún está vigente para realizar la verificación de información; esto hace las veces de la sección presentada con un evento de captura de tiempo y un evento de recepción de mensaje.
También, por la falta de compuertas con varios flujos incidentes y varios flujos salientes, fue necesario el uso de compuertas XOR para las secciones en las que convergen varios flujos y salen varios flujos.

Se implementó una tarea script ubicada entre el proceso multiinstancia y la actividad de publicitar admisión, esto con el fin de instanciar datos de prueba provisionales, ya que no fue posible tener acceso a la base de datos requerida.

Otra tarea de script fue implementada posteriormente a la tarea de envío del archivo plano, ya que las notificaciones proveídas por la suite no tienen la capacidad de enviar archivos adjuntos, por lo que la tarea de envío solo captura la ruta del archivo y en la de script se realiza el envío como tal. 


\section{E. Evaluación de las herramientas}

Se maneja una metodología de evaluación basada en ponderaciones y gradaciones $[10,11]$ de parámetros, factores e índices, definidos y calificados bajo criterio del evaluador. Las escalas correspondientes se pueden observar en las tablas 3 y 4.

TABLA 3

ESCALA DE PONDERACIÓN [10]

\begin{tabular}{|l|c|}
\hline \multicolumn{1}{|c|}{ Nivel de importancia } & Valoración \\
\hline Indispensable & 9 a 10 \\
\hline Necesario & 7 a 8 \\
\hline Importante & 4 a 6 \\
\hline Complementario & 1 a 3 \\
\hline
\end{tabular}

TABLA 4

ESCALA DE GRADACIÓN [10]

\begin{tabular}{|l|c|}
\hline Nivel de importancia & Gradación \\
\hline Deficiente & 0.0 hasta 0.9 \\
\hline Insuficiente & 1.0 hasta 1.9 \\
\hline Regular & 2.0 hasta 2.9 \\
\hline Aceptable & 3.0 hasta 3.9 \\
\hline Bueno & 4.0 hasta 4.6 \\
\hline Excelente & 4.7 hasta 5.0 \\
\hline
\end{tabular}

Para evaluar cada aspecto se utilizan las ecuaciones, que deben utilizarse en el orden respectivo:

$$
\begin{gathered}
\text { Gradación Factor }=\left(\frac{\sum(\text { Ponderación Índice*Gradación Índice })}{\sum(\text { Ponderación Índice } * 5)}\right) * 5 \\
\text { Gradación Parámetro }=\left(\frac{\sum(\text { Ponderación Factor } * \text { Gradación Factor })}{\sum(\text { Ponderación Factor } * 5)}\right) * 5 \\
\text { Resultado Fin Parámetro }=\left(\frac{\sum(\text { Ponderación Parámetro } * \text { Gradación Parámetro })}{\sum(\text { Ponderación Parámetro } * 5)}\right) * 5
\end{gathered}
$$

Después de aplicar estas ecuaciones y de obtener una calificación final de la herramienta en los parámetros evaluados, se procede a realizar una calificación de la documentación y soporte proveída por cada herramienta; esto debido a que este aspecto es transversal a todos los parámetros evaluados, y por esto debería tener una ponderación dentro de la evaluación realizada a las herramientas. Para el caso particular se consideró que la calificación de los parámetros representaría un $90 \%$ de la evaluación final, y la documentación y soporte, un $10 \%$, lo que lleva a aplicar (1).

$$
\text { Evaluación Final }=(\text { ResultadoFin.Parám. } * 0.90)+(\text { EvaluaciónDoc. } * 0.10)
$$

Con (4) se obtendrá una evaluación final que determinará qué herramienta es más apta de acuerdo con el caso de estudio.

\section{Resultados}

A continuación se enmarcan los resultados obtenidos para la evaluación de los parámetros, factores e índices seleccionados para la evaluación de la herramienta. 
TABLA 5

PONDERACIÓN Y GRADACIÓN DE LA IMPLEMENTACIÓN BPM

\begin{tabular}{|c|c|c|c|c|c|c|c|}
\hline \multirow{2}{*}{ Parámetro } & \multirow{2}{*}{ Pond. } & \multirow{2}{*}{ Factor } & \multirow{2}{*}{ Pond. } & \multirow{2}{*}{ Índice } & \multirow{2}{*}{ Pond. } & \multicolumn{2}{|c|}{$\begin{array}{l}\text { Gradación del } \\
\text { índice }\end{array}$} \\
\hline & & & & & & $\begin{array}{l}\text { Oracle } \\
\text { BPM }\end{array}$ & JBPM \\
\hline \multirow{16}{*}{$\begin{array}{l}\text { Implementación } \\
\text { BPM }\end{array}$} & \multirow{16}{*}{10} & \multirow{4}{*}{$\begin{array}{l}\text { Configuración general de un } \\
\text { proyecto }\end{array}$} & \multirow{4}{*}{8} & $\begin{array}{l}\text { Creación de un } \\
\text { proyecto }\end{array}$ & 8 & 4,30 & 4,50 \\
\hline & & & & Diseño de un proceso & 10 & 4,70 & 4,80 \\
\hline & & & & $\begin{array}{l}\text { Despliegue de un } \\
\text { proceso }\end{array}$ & 10 & 3,50 & 3,40 \\
\hline & & & & $\begin{array}{l}\text { Ejecución de un pro- } \\
\text { ceso }\end{array}$ & 10 & 3,50 & 4,80 \\
\hline & & Interfaz gráfica & 7 & $\begin{array}{l}\text { Fácil entendimiento de } \\
\text { interfaz gráfica }\end{array}$ & 7 & 3,80 & 4,20 \\
\hline & & \multirow{2}{*}{$\begin{array}{l}\text { Gestión de iconos de la nota- } \\
\text { ción para el diseño }\end{array}$} & \multirow[t]{2}{*}{8} & $\begin{array}{l}\text { Representación gráfica } \\
\text { adecuada del compo- } \\
\text { nente }\end{array}$ & 6 & 4,30 & 4,30 \\
\hline & & & & $\begin{array}{l}\text { Clasificación de los } \\
\text { componentes }\end{array}$ & 5 & 4,50 & 4,50 \\
\hline & & \multirow{2}{*}{$\begin{array}{l}\text { Implementación de pantallas de } \\
\text { usuario }\end{array}$} & \multirow{2}{*}{9} & $\begin{array}{l}\text { Personalización de } \\
\text { pantallas }\end{array}$ & 7 & 4,00 & 4,20 \\
\hline & & & & $\begin{array}{l}\text { Asociación de } \\
\text { pantallas a actividades }\end{array}$ & 9 & 3,70 & 4,10 \\
\hline & & \multirow{3}{*}{ Implementación de eventos } & \multirow{3}{*}{8} & $\begin{array}{l}\text { Implementación de } \\
\text { eventos de captura }\end{array}$ & 6 & 3,90 & 4,30 \\
\hline & & & & $\begin{array}{l}\text { Implementación de } \\
\text { eventos de inicio y fin }\end{array}$ & 8 & 4,50 & 4,50 \\
\hline & & & & $\begin{array}{l}\text { Implementación de } \\
\text { eventos de interrup- } \\
\text { ción }\end{array}$ & 8 & 4,70 & 3,90 \\
\hline & & $\begin{array}{l}\text { Modificación al proceso origi- } \\
\text { nal para implementación en la } \\
\text { herramienta }\end{array}$ & 8 & & & & \\
\hline & & Implementación de actividades & 10 & & & & \\
\hline & & Implementación de compuertas & 10 & & & & \\
\hline & & $\begin{array}{l}\text { Implementación de } \\
\text { notificaciones }\end{array}$ & 5 & & & & \\
\hline
\end{tabular}

TABLA 6

PONDERACIÓN Y GRADACIÓN DE LA INTEGRACIÓN

\begin{tabular}{|c|c|c|c|c|c|c|c|}
\hline \multirow{2}{*}{ Parámetro } & \multirow{2}{*}{ Pond. } & \multirow{2}{*}{ Factor } & \multirow{2}{*}{ Pond. } & \multirow{2}{*}{ Índice } & \multirow{2}{*}{ Pond. } & \multicolumn{2}{|c|}{ Gradación del índice } \\
\hline & & & & & & Oracle BPM & JBPM \\
\hline \multirow[t]{7}{*}{ Integración } & \multirow[t]{7}{*}{7} & \multirow[t]{3}{*}{ Integración de protocolos } & \multirow[t]{3}{*}{8} & Integración HTTP & 9 & 4,70 & 4,70 \\
\hline & & & & Integración JMS & 9 & 4,50 & 4,00 \\
\hline & & & & Integración FTP & 4 & 4,50 & 3,20 \\
\hline & & Integración de webservices & 9 & & & & \\
\hline & & $\begin{array}{l}\text { Envío de mensajes entre pro- } \\
\text { cesos }\end{array}$ & 10 & & & & \\
\hline & & Integración con bases de datos & 10 & & & & \\
\hline & & Integración con frameworks & 8 & & & & \\
\hline
\end{tabular}


TABLA 7

PONDERACIÓN Y GRADACIÓN DE LA ESCALABILIDAD

\begin{tabular}{|c|c|l|c|}
\hline Parámetro & Pond. & \multicolumn{1}{|c|}{ Factor } & Pond. \\
\hline \multirow{2}{*}{ Escalabilidad } & \multirow{2}{*}{6} & Uso de recursos de hardware & 9 \\
\cline { 3 - 4 } & & Mejora de procesos & 10 \\
\hline
\end{tabular}

TABLA 8

PONDERACIÓN Y GRADACIÓN DEL DESEMPEÑO

\begin{tabular}{|c|c|l|c|}
\hline Parámetro & Pond. & \multicolumn{1}{c|}{ Factor } & Pond. \\
\hline \multirow{4}{*}{ Desempeño } & \multirow{4}{*}{9} & Robustez & 7 \\
\cline { 3 - 4 } & \multirow{2}{*}{} & Latencia & 7 \\
\cline { 3 - 4 } & & Productividad & 9 \\
\cline { 3 - 4 } & & Gestión de roles & 10 \\
\cline { 3 - 4 } & Eficacia & 10 \\
\hline
\end{tabular}

En la tabla 9 se presenta el consolidado de comparación de las dos herramientas, después de aplicar (1) sobre los índices para la obtención de una gradación de factores; además de presentar los parámetros y factores para los que no fueron definidos índices de evaluación.

TABLA 9

GRADACIÓN DE FACTORES

\begin{tabular}{|c|c|c|c|c|c|}
\hline \multirow{2}{*}{ Parámetro } & \multirow{2}{*}{ Pond. } & \multirow{2}{*}{ Factor } & \multirow{2}{*}{ Pond. } & \multicolumn{2}{|c|}{ Gradación del factor } \\
\hline & & & & Oracle BPM & JBPM \\
\hline \multirow{9}{*}{$\begin{array}{l}\text { Implementación } \\
\text { BPM }\end{array}$} & \multirow{9}{*}{10} & Configuración general de un proyecto & 8 & 3,98 & 4,37 \\
\hline & & Interfaz gráfica & 7 & 3,80 & 4,20 \\
\hline & & Gestión de iconos de la notación para el diseño & 8 & 4,39 & 4,39 \\
\hline & & Implementación de pantallas de usuario & 9 & 3,83 & 4,14 \\
\hline & & Implementación de eventos & 8 & 4,41 & 4,23 \\
\hline & & $\begin{array}{l}\text { Modificación al proceso original para implementación en } \\
\text { la herramienta }\end{array}$ & 8 & 4,80 & 3,80 \\
\hline & & Implementación de actividades & 10 & 4,80 & 4,00 \\
\hline & & Implementación de compuertas & 10 & 4,20 & 4,20 \\
\hline & & Implementación de notificaciones & 5 & 4,90 & 3,50 \\
\hline \multirow{5}{*}{ Integración } & \multirow{5}{*}{7} & Integración de protocolos & 8 & 4,58 & 4,14 \\
\hline & & Integración de webservices & 9 & 4,10 & 4,00 \\
\hline & & Envío de mensajes entre procesos & 10 & 4,70 & 3,70 \\
\hline & & Integración con bases de datos & 10 & 4,00 & 4,80 \\
\hline & & Integración con frameworks & 8 & 4,00 & 4,40 \\
\hline \multirow{2}{*}{ Escalabilidad } & \multirow{2}{*}{6} & Uso de recursos de hardware & 9 & 3,50 & 4,30 \\
\hline & & Mejora de procesos & 10 & 4,00 & 4,00 \\
\hline \multirow{5}{*}{ Desempeño } & \multirow{5}{*}{9} & Robustez & 7 & 4,40 & 3,60 \\
\hline & & Latencia & 7 & 4,20 & 4,20 \\
\hline & & Productividad & 9 & 4,00 & 3,80 \\
\hline & & Gestión de roles & 10 & 4,30 & 4,40 \\
\hline & & Eficacia & 10 & 4,40 & 4,40 \\
\hline
\end{tabular}


Luego, se aplica (2), gradación sobre los factores, y se obtiene una tabla (ver tabla 10) con los parámetros y sus respectivos valores de gradación.

\section{TABLA 10}

GRADACIÓN DE PARÁMETROS

\begin{tabular}{|l|c|c|c|}
\hline \multirow{2}{*}{ Parámetro } & \multirow{2}{*}{ Ponderación } & \multicolumn{2}{c|}{$\begin{array}{c}\text { Gradación del } \\
\text { parámetro }\end{array}$} \\
\cline { 3 - 4 } & & $\begin{array}{c}\text { Oracle } \\
\text { BPM }\end{array}$ & JBPM \\
\hline $\begin{array}{l}\text { Implementa- } \\
\text { ción BPM }\end{array}$ & 10 & 4,33 & 4,12 \\
\hline Integración & 7 & 4,28 & 4,20 \\
\hline Escalabilidad & 6 & 3,76 & 4,14 \\
\hline Desempeño & 9 & 4,26 & 4,11 \\
\hline
\end{tabular}

Finalmente, para obtener una calificación y consolidación general de las herramientas y lograr una evaluación acertada sobre ellas se procede a aplicar (3) sobre los parámetros (ver tabla 11).

\section{TABla 11}

VALORACIÓN FINAL DE LAS HERRAMIENTAS EN LOS PARÁMETROS

\begin{tabular}{|c|c|}
\hline Oracle BPM & JBPM \\
\hline 4,19 & 4,13 \\
\hline
\end{tabular}

Luego de la evaluación de las herramientas en los parámetros acordados se procede a dar una calificación a la documentación y soporte en cada herramienta, con el fin de obtener la evaluación final de cada una de ellas. Esta calificación se presenta en la tabla 12.

\section{TABLA 12}

CALIFICACIÓN DADA PARA DOCUMENTACIÓN Y SOPORTE

\begin{tabular}{|c|c|}
\hline Oracle BPM & JBPM \\
\hline 2,30 & 4,00 \\
\hline
\end{tabular}

Teniendo las calificaciones de parámetros y de documentación y soporte se procede a calcular la evaluación final, presentada en la tabla 13.
TABLA 13

EVALUACIÓN FINAL DE LAS HERRAMIENTAS

\begin{tabular}{|c|c|}
\hline Oracle BPM & JBPM \\
\hline 4,00 & 4,11 \\
\hline
\end{tabular}

Después de realizar las diferentes ponderaciones, de comparar las herramientas en cada de sus características que se tomaron para este trabajo y de obtener la evaluación final, se puede observar que JBPM cuenta con un mejor desempeño en la automatización del proceso de admisiones.

\section{Conclusiones}

La evaluación cuantitativa por parámetros brinda una mejor visión de la funcionalidad de un software, especialmente por basarse en el criterio del usuario, quien es la persona adecuada para juzgarlo, pues conoce el objetivo específico por el cual se desea usar una herramienta.

Las metodologías iterativas ágiles de desarrollo desoftware son las más adecuadas para desarrollar aplicaciones basadas en procesos con BPM, debido a que ofrecen la rapidez que requiere este último para mejorar la productividad y eficacia de los procesos organizacionales.

\section{REFERENCIAS}

[1] J. A. Villasís, T. Gualotuña y C. Hinojosa, Metodología para el análisis, diseño e implementación de procesos con tecnología BPM y desarrollo de un caso práctico [Online] Available: http://repositorio.espe. edu.ec/handle/21000/6298. [Accessed: 18.mar-2013].

[2] F. N. Díaz, Gestión de Procesos de Negocio BPM (Business Process Management), TICs y Crecimiento Empresarial. ¿Qué es BPM y cómo se articula con el crecimiento empresarial? Revista Universidad \& Empresa, Vol. 15, pp. 151-176, Oct. 2008.

[3] S. White y D. Miers, BPMN Guía de Referencia y Modelado. Future Strategies Inc, 2009. 
[4] Oracle Business Process Management | Oracle. [Online]. Available: http://www.oracle.com/ lad/technologies/bpm/index.html. [Accessed: 21-abr-2012].

[5] Business Process Management Suite - Features \& Benefits | Oracle. [Online]. Available: http://www.oracle.com/us/technologies/bpm/ suite/features/index.html. [Accessed: 21-abr2012].

[6] jBPM-JBoss Community [Online]. Available: $\mathrm{http} / / /$ www.jboss.org/jbpm. [Accessed: 15jun-2012].

[7] Processes, Rules and Events: jBPM 5.2 released [Online]. Available: http://kverlaen. blogspot.com/2011/12/jbpm-52-released. html. [Accessed: 15-jun-2012].
[8] D. Mamani, Metodología Scrum, Gestión ágil de proyectos. Universidad Unión Bolivariana, 2009.

[9] J. Palacio, Flexibilidad con Scrum, Principios de diseño e implantación de campos de Scrum. oct-2008. Available: http://www.safecreative. org/work/0710210187520

[10] C. Suárez. Estudio comparativo de herramientas desktop gis libres Udig vs. Mapserver". Universidad Pedagógica y Tecnológica de Colombia, Facultad de Ingeniería. 2010.

[11] J. S. González and G. Cáceres. "Comparasion of GIS Desktop Tools for Development of SIGPOT". IEEE Latin America Transactions, Vol. 11, No. 1, pp. 86-90, Feb. 2013. 